\section{Psychopathologies of Social Cognition: Linking Neural, Cognitive and Clinical Perspectives}

\section{R Langdon}

Macquarie Centre for Cognitive Science, Macquarie University, New South Wales, Australia

\section{Overview}

Social cognition encompasses the processing of affective and cognitive mental states of self and other. A number of psychopathologies have now been linked to abnormalities of social cognition prompting questions about interactions and dissociations within the sociocognitive domain and highlighting gaps in the literature. This symposium begins with an overview of historical beginnings, theoretical accounts and questions concerning neural underpinnings. Individual speakers will then focus on different psychopathologies to present reviews and some new empirical data to address unresolved issues, highlight new directions and offer cautionary notes concerning the conduct of social cognition research in clinical populations.

06-01

\section{Emotion regulation in affective and nonaffective psychoses}

\section{MJ Green ${ }^{1,2}$}

'School of Psychiatry, University of New South Wales, Sydney, New South Wales, Australia; and 'Black Dog Institute, Prince of Wales Hospital, Sydney, New South Wales, Australia

Background: Emotion regulation involves the cognitive manipulation of subjective and physiological experiences of emotion. Recent neuroimaging studies implicate distinct patterns of prefrontal cortical inhibition of subcortical regions in association with particular cognitive regulatory strategies, alongside modulation of autonomic responses associated with various forms of negative affect. The use of maladaptive cognitive regulatory mechanisms is associated with altered neural activity in healthy individuals and increased negative affect in healthy and clinical populations.

Methods: The role of emotion dysregulation in the development and maintenance of affective and psychotic symptoms is considered from a cognitive neuroscience perspective. Neuropsychological and social-cognitive processes involved in emotion regulation are discussed in the context of neural mechanisms of cognitive con- trol and emotion processing in healthy individuals. The symptoms of affective (bipolar, schizoaffective) and nonaffective (schizophrenia) psychotic disorders are considered as manifestations of emotion dysregulation according to known neuropsychological and social processing deficits, and clinical neuropathology.

Results: Emotion regulation relies on synergy within bidirectional fronto-striatal-thalamic and brainstem networks involved in emotion perception, affect generation and control of the autonomic nervous system. Convergent evidence from cognitive neuropsychological and neurobiological investigations in affective and nonaffective psychoses implicates differential dysfunction in these neural systems supporting the cognitive regulation of emotion.

Conclusions: The cognitive control of emotion may be disrupted by abnormalities in cognitive and/or neural processes subserving social perception, affect generation or regulation. Consideration of the neuropsychological and social-cognitive profiles of affective and nonaffective psychoses alongside regional neuropathology suggests the existence of distinct patterns of emotion dysregulation in these conditions.

06-02

\section{Processing and responding to social stimuli in borderline personality disorder: a selective review}

\section{Jovev}

ORYGEN Research Centre, Department of Psychiatry, The University of Melbourne, Melbourne, Australia

Background: Dysfunction in emotional systems has long been emphasized in the theories of borderline personality disorder (BPD); however, there has been a paucity of research exploring the relationship between emotion dysfunction and social functioning in this disorder.

Methods: This paper reports a selective review of the literature on processing and responding to social stimuli in BPD. The paper begins with a general review of the role of emotion and social behaviour in psychopathology and then focuses more specifically on research in various domains of social cognition and attention in BPD. The neuroanatomical regions that have been implicated in studies of social cognition and BPD are also discussed.

Results: The integration of results from studies of emotional, neurocognitive and social dysfunctions in BPD can help to identify potential origins and social consequences of BPD and define the way in which various social functions of emotion processing are shaped 